\title{
A two-step method to make microglia
}

Microglia have been reported in some disease models to have beneficial effects; however, research into their potential as a cell therapy is limited by the lack of means to produce readily grafted, autologous microglial cells. Now, in Nature Neuroscience, Pandya et al. describe a method for differentiating human and murine induced pluripotent stem cells (iPSCs) into microglia-like cells (iPS-MGs).

The authors used a two-step method to reprogramme two human iPSC lines. First, the iPSCs were converted into haematopoietic progenitor-like cells (HPCs) either through co-culture with OP-9 cells (mouse stromal cells) or by incubation in differentiation medium supplemented with various growth factors. Second, the resulting HPCs - which expressed the HPC markers CD34, CD43 and CD45 - were co-cultured with human astrocytes for 2 weeks in medium containing granulocytemacrophage colony-stimulating factor (CSF), macrophage CSF and IL-3. At the end of this period, immunostaining revealed that many of the cells expressed markers characteristic of microglia, including IBA1 and CD39.

The authors compared the gene expression signatures of the iPS-MGs with those of the original iPSC lines, as well as those of dendritic cells, macrophages and human fetal microglia. Correlation-based cluster analysis revealed that the gene expression signature of the iPS-MGs resembled that of the human fetal microglia, and quantitative reverse transcription PCR analysis confirmed that the iPS-MGs express the consensus gene signature characteristic of microglia. Moreover, the iPS-MGs were able to phagocytose fluorescently labelled bacteria and, upon immune stimulation, produced reactive oxygen species (ROS) and tumour necrosis factor. Thus, the iPS-MGs show similar gene expression profiles and functions to microglia.

Next, the authors generated murine iPSCs from mice expressing green fluorescent protein (GFP) under the control of the $C \times 3 \mathrm{cr} 1$ promoter $\left(\mathrm{C} x 3 \mathrm{cr} 1^{\mathrm{GFP} / \mathrm{t}}\right.$ mice $)$ and converted these cells into murine iPS-MGs using the same two-step protocol as for one of the human iPSC lines. Similar to the human iPS-MGs, murine iPS-MGs showed a similar gene expression profile to that of murine microglia, and were able to phagocytose bioparticles and produce ROS.

iPS-MGs derived from $C \times 3 c r 1^{\mathrm{GFP} /+}$ mice were implanted into the left cerebral hemispheres of recipient mice bearing glioma tumours in the right cerebral hemisphere. One week later, $\mathrm{IBA}^{+} \mathrm{GFP}^{+}$cells were observed in the tumour site, indicating that these iPS-MGs - like normal microglia - migrate towards glioma.

Last, the authors tested the effects of implanted immune cells that were previously exposed to tumour cell lysate in vitro on the survival of mice with intracranial glioma. Specifically, they tested the effects of intracranial implantation of mouse neonate microglia or murine iPS-MGs in combination with subcutaneously implanted dendritic cells (DCs). Control mice that received no cell implants had a median survival of
40 days, whereas half of the mice that received a combination of DCs and either mouse neonate microglia or murine iPS-MGs survived to the end of the 1-year assessment period. This result indicates that iPS-MGs, like endogenous microglia, could have antitumour functions. These antitumour actions could involve presenting tumour antigens and/or directly killing glioma cells.

This study demonstrates a method for generating iPS-MGs that could prove useful for toxicity-screening and drug-screening assays, as well as for investigation into the possible therapeutic potential of microglia.

\section{Natasha Bray}

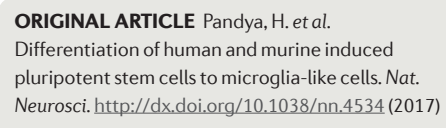

iPS-MGs show

similar gene

expression

profiles and

functions to

microglia

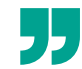

